Full length article

\title{
Which is the best predictor of excessive hip internal rotation in women with patellofemoral pain: Rearfoot eversion or hip muscle strength? Exploring subgroups
}

\author{
Amanda Schenatto Ferreira ${ }^{\mathrm{a}}$, Danilo de Oliveira Silva ${ }^{\mathrm{a}, \mathrm{b}, 1}$, Ronaldo Valdir Briani ${ }^{\mathrm{a}}$, Deisi Ferrari ${ }^{\mathrm{a}}$, \\ Fernando Amâncio Aragão $^{c}$, Marcella Ferraz Pazzinatto ${ }^{\mathrm{a}, \mathrm{b}}$, Fábio Mícolis de Azevedo, ${ }^{\mathrm{a}, *}$

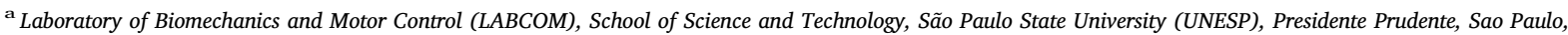 \\ Brazil \\ ${ }^{\mathrm{b}}$ La Trobe Sports and Exercise Medicine Research Centre (LASEM), School of Allied Health, La Trobe University, Bundoora, Victoria, Australia \\ ${ }^{\mathrm{c}}$ Laboratory of Human Movement Research (LAPEMH), State University of West Parana, Cascavel, Parana, Brazil
}

\section{A R T I C L E I N F O}

\section{Keywords:}

Anterior knee pain

Hip

Knee

Foot

Kinematics

Strength

\begin{abstract}
A B S T R A C T
Background: Patellofemoral pain (PFP) has been linked to increased patellofemoral joint stress as a result of excessive hip internal rotation. Lower hip strength and/or excessive rearfoot eversion have been used to explain such altered movement pattern; however, it is unknown which one is the best predictor of excessive hip internal rotation.

Research question: To investigate if peak rearfoot eversion and/or peak concentric hip abductor strength can predict peak hip internal rotation during stair ascent in women with PFP.

Methods: This cross-sectional study included thirty-seven women with PFP which underwent three-dimensional kinematic analysis during stair ascent and hip abductor strength analysis in an isokinetic dynamometer. A forced entry linear regression model analysis was carried out to determine which independent variables present the best capability to predict the hip internal rotation.

Results: Peak concentric hip abductor strength significantly predicted peak hip internal rotation during stair ascent $\left(\mathrm{R}^{2}=0.27, \mathrm{p}=0.001\right)$. Peak rearfoot eversion did not predict peak hip internal rotation during stair ascent $\left(\mathrm{R}^{2}<0.01, \mathrm{p}=0.62\right)$. A Post-hoc analysis was conducted to explore if a subgroup with excessive rearfoot eversion would predict hip internal rotation. Based on a previous reported cut-off point, $48.6 \%$ of the participants were classified as excessive rearfoot eversion. For the subgroup with excessive rearfoot eversion, peak concentric hip abductor strength and peak rearfoot eversion significantly predicted peak hip internal rotation during stair ascent $\left(\mathrm{R}^{2}=0.26, \mathrm{p}=0.02 ; \mathrm{R}^{2}=0.42, \mathrm{p}=0.003\right.$, respectively). For non-excessive rearfoot eversion subgroup, peak concentric hip abductor strength significantly predicted peak hip internal rotation during stair ascent $\left(\mathrm{R}^{2}=0.53 ; \mathrm{p}<0.001\right)$; and peak rearfoot eversion did not $\left(\mathrm{R}^{2}=0.01 ; \mathrm{p}=0.65\right)$.

Significance: Findings indicate that hip muscle strength seems to be related with hip internal rotation in all women with PFP. Rearfoot eversion seems to be related with hip internal rotation only in a subgroup with excessive rearfoot eversion.
\end{abstract}

\section{Introduction}

Patellofemoral pain (PFP) is a common complaint amongst sports medicine clinics, accounting for $25-40 \%$ of all knee injuries [1]. It has been reported that women are more likely to experience this condition than men [2]. Clinically, individuals with PFP report limitations in activities that load the patellofemoral joint (PFJ) during weight bearing on a flexed knee [3], especially ascending stairs [4]. Furthermore, the impact of PFP as a medical issue is highlighted by evidence suggesting a chronic and recurrent nature of pain, persisting for many years with unfavorable outcomes after rehabilitation [5].

The source of pain in individuals with PFP remains unclear [3].

\footnotetext{
* Corresponding author at: São Paulo State University (UNESP), School of Science and Technology, Physical Therapy Department, "Laboratory of Biomechanics and Motor Control (LABCOM)", Rua Roberto Simonsen, 305 Presidente Prudente, Sao Paulo, Postal Code (CEP): 19060-900, Brazil.

E-mail addresses: amandaschenatto_@outlook.com (A.S. Ferreira), danilo110190@hotmail.com (D. de Oliveira Silva), ronaldobriani@hotmail.com (R.V. Briani), deisiferrari@hotmail.com (D. Ferrari), feraaragao@gmail.com (F.A. Aragão), ferraz_mar@hotmail.com (M.F. Pazzinatto), micolis@fct.unesp.br (F.M. de Azevedo).

${ }^{1}$ Present address: La Trobe University, School of Allied Health, Bundoora, Victoria, Australia.
} 
However, the most recent consensus statement from the 4th international PFP retreat proposed a pathomechanical model in which the development of PFP is associated with increased PFJ stress. One of the theories indicate excessive hip internal rotation during weight bearing activities as a possible source of increased PFJ stress [6-8]. There are two well-known theoretical models which attempt to explain the cause of excessive hip internal rotation in women with PFP [9,10]. One assumes that the hip joint depends on hip muscles to provide dynamic stability and control the movement of the femur, therefore, reduced hip strength may lead to a poor hip control and result in an excessive hip internal rotation [9]. Lower isometric, concentric and eccentric hip abductor strength have been reported in women with PFP [11-13]. Specifically, lower concentric hip abductor strength may be associated with excessive hip internal rotation during stair ascent. In this activity, predominantly concentric contractions of hip abductors are required to control the hip movement $[14,15]$. Another theoretical model assumes that excessive rearfoot eversion during the stance phase of gait [16] or stair ascent [4] may result in excessive hip internal rotation due to joint coupling [10].

Considering the need to reduce PFJ stress, knowing the mechanism that is more associated with hip internal rotation could help clinicians to identify the best treatment strategy to individuals with PFP. For instance, if the lower hip abductor strength is more associated with hip internal rotation, strengthening the hip abductor muscles could be an option to avoid increased PFJ stress in women with PFP. On the other hand, if the excessive rearfoot eversion is more associated, foot orthoses prescription could be also performed in order to avoid the increased PFJ stress [17].

In this context, the aim of this study was: (i) to investigate if the peak rearfoot eversion during stair ascent and/or the peak concentric abductor muscle strength can predict peak hip internal rotation during stair ascent in women with PFP.

\section{Methods}

\subsection{Participants}

Thirty-seven women with PFP aged 18 to 35 years were recruited between September 2016 and May 2017 via advertisements at fitness centers, public places for physical activity and social medias. The study was approved by the Local Human Ethics Committee, and all participants provided written informed consent. The diagnostic criteria for PFP is listed in Table 1.

\subsection{Procedures}

Demographic data (age, body mass and height) were collected prior

Table 1

Inclusion and exclusion criteria for PFP.

Inclusion criteria

Insidious onset symptoms lasting at least 4 months

Worst pain level in the previous month corresponding to at least $30 \mathrm{~mm}$ in the visual analogue pain scale (VAS)

Anterior knee pain when performing at least two of the following activities: sitting for prolonged time, squatting, kneeling, running, ascending and descending stairs, jumping and landing

Exclusion criteria

Sign or symptoms of any other knee dysfunction

History of surgery in any lower limb joint

History of patellar subluxation

Clinical evidence of meniscal injury or ligament instability

Referred pain coming from the lumbar spine.

Note: The eligibility criteria were assessed by a clinician ( $>5$ years of clinical experience) based on previous studies[3,4]. to testing. All participants were asked to rate their worst knee pain intensity during the last month, and current pain measured on a 0-100 visual analogue scale (VAS). Additionally, duration of knee-related symptoms (months) and self-reported knee function (anterior knee pain scale - AKPS) were obtained.

Kinematic and isokinetic assessment were performed on two different days with interval of $48 \mathrm{~h}$ to one week to prevent any possible influence of neuromuscular fatigue or muscle soreness. The order of the assessments was randomized to avoid systematic bias. Data collection was performed in participant's symptomatic limb (unilateral symptoms) or most symptomatic limb (bilateral symptoms) [4,16]. All assessments were conducted by the same evaluator.

\subsection{Kinematic assessment}

The kinematic assessment was performed during a stair ascent task. Kinematic data was collected using a three-dimensional motion analysis system (Vicon Motion Systems Inc.; Denver EUA) combined with 9 cameras (type Bonita ${ }^{\circ} \mathrm{B} 10$ ) operating at a sampling frequency of $100 \mathrm{~Hz}$ with a resolution of 1 megapixel. Ground reaction forces were collected using a force plate (Bertec Corporation, Columbus, OH, model FP4060) at a sampling frequency of $4000 \mathrm{~Hz}$. The force plate and motion system were synchronized by the Vicon Lock device.

Retroreflective markers $(9.5 \mathrm{~mm})$ were placed on the participants in accordance with the Oxford Foot Model (OFM) combined with plug-in gait (PiG to lower limbs), which was previously reported as a valid and reliable approach $[16,18,19]$. In addition to these models, three markers were inserted (center of the patella, anterior thigh and medial knee) in order to improve the acquisition accuracy of the hip internal rotation angles. The anatomical landmarks are described in Appendix A as well as the reliability and accuracy data of a pilot study conducted with 12 participants.

A relaxed standing calibration trial was then captured, after that the participants performed three practice stair ascent trials to allow familiarization with the instrumentation and environment. Each participant was asked to climb a seven-step staircase (each step being $18 \mathrm{~cm}$ high and $28 \mathrm{~cm}$ deep [Fig. 1A]) at their natural comfortable speed [20]. The force plate was mechanically coupled to the ground (i.e. independent and uncoupled from the stair structure) (Fig. 1B) [4]. Five successful trials were collected for each participant and the mean value of these five trials was used for data analyses in order to attenuate the influence of speed, intra-subject variability, among other external factors. A trial was considered successful when the tested limb touched the fourth step (where force plate was allocated) and the participant has performed the stair ascent with consistent velocity (i.e., at their natural comfortable speed during five trials). We found an average of 0.12 unsuccessful trials. In the case of unsuccessful trials, an additional trial was performed.

\subsection{Isokinetic assessment}

Concentric torque of the hip abductors was assessed using an isokinetic dynamometer (Biodex System 4 Pro, New York, USA). Concentric contractions were evaluated as during stair ascent task hip muscles act mainly concentrically to perform the movement $[14,15]$. The isokinetic assessment was performed using a previous used protocol $[12,13]$ which is described in detail in the Appendix B.

\subsection{Data analysis}

All kinematic data were filtered with a fourth-order Butterworth low-pass filter with a cutoff frequency of $6 \mathrm{~Hz}$ labeled and reconstructed within the Vicon Nexus ${ }^{\infty}$ software. The vertical ground reaction force signals were used to identify the stance phase from which the measurements of interest were computed [4]. The cadence was calculated based on the time of one entire gait cycle: single leg stance between toe- 


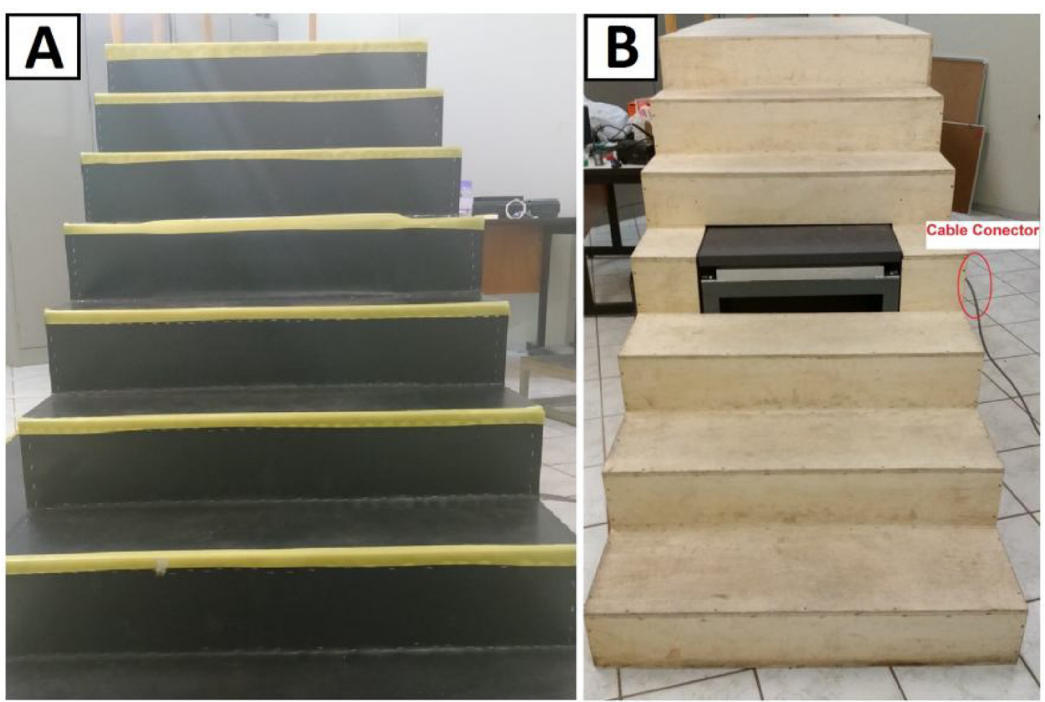

Fig. 1. A) The seven-step stair-case used in data collection. B) The force plate was mechanically coupled to the ground (i.e. independent and uncoupled from the stair structure).

off of the opposite leg from the third step until foot contact on the fifth step. Each event was inspected manually by viewing the animated visualization of the motion data [4].

The kinematic and isokinetic data were analyzed using a custom code in MatLab (MATLAB; The MathWorks, Inc, Natick, MA). The kinematics variables of interest were peak angles of hip internal rotation and rearfoot eversion during stance phase. The isokinetic variable of interest was peak concentric hip abductor strength, measured as the average of the middle 3 repetitions for each strength test to eliminate the possibility of learning (trial 1) and fatigue effects (trial 5) [13]. All torque data $(\mathrm{Nm})$ were normalized by body mass $([\mathrm{Nm} / \mathrm{kg}] \times 100)$ [12].

\subsection{Statistical analysis}

We performed a sample size calculation for multiple regression models that revealed a minimum sample size of 31 participants per group to detect a large effect size (0.35), with alpha set at 0.05 and power of $80 \%$.

A forced entry linear regression model analysis was carried out to determine which independent variables present the best capability to predict the hip internal rotation. The variables were inserted into the model separately to distinguish which was more predictive. The assumption of homogeneity of variance and linearity was verified. The correlation coefficients of each independent variable and the dependent variable were identified and the performance of the model was evaluated using $\mathrm{R}^{2}$, which estimates explained variation of the model. Statistical analyses were performed using the Statistical Software for Social Sciences (IBM 23.0, SPSS inc., Chicago, IL) with an a priori level of significance of 0.05 .

\section{Results}

Descriptive values of participant's demographics, self-reported measures of pain, function and duration of symptoms, kinematics and strength variables are presented in Table 2 .

Peak concentric hip abductor strength significantly predicted peak hip internal rotation during stair ascent $\left(\mathrm{R}^{2}=0.27, \mathrm{~B}=-0.05,95 \% \mathrm{CI}\right.$ -0.08 to $-0.02, p=0.001)$. Peak rearfoot eversion did not predict peak hip internal rotation during stair ascent $\left(\mathrm{R}^{2}<0.01, \mathrm{~B}=0.12\right.$, 95\%CI -0.40 to $0.65, \mathrm{p}=0.62$ ) (Fig. 2).

\subsection{Post hoc analysis}

Based on non-significant findings of peak rearfoot eversion vs. peak hip internal rotation and suggestions during the peer review. We have performed a post hoc analysis to further explore this relationship in a specific subgroup of women with PFP with excessive rearfoot eversion. Previously published cut-off points [21] were used to split the sample into excessive and non-excessive rearfoot eversion subgroups. Linear and multiple regressions analysis were then performed to determine which independent variables present the best capability to predict the hip internal rotation in each subgroup. Considering the cut-off points, $48.6 \%$ of women with PFP were classified as excessive rearfoot eversion. Descriptive values of self-reported pain and function, kinematics and strength variables for each subgroup are reported in Table 2 .

For excessive rearfoot eversion subgroup, linear regression models demonstrated that peak concentric hip abductor strength significantly predicted peak hip internal rotation during stair ascent $\left(\mathrm{R}^{2}=0.26\right.$, $\mathrm{B}=-0.22,95 \% \mathrm{CI}-0.43$ to $-0.02, \mathrm{p}=0.02$ ); and peak rearfoot eversion significantly predicted peak hip internal rotation during stair ascent $\left(\mathrm{R}^{2}=0.42, \mathrm{~B}=3.19,95 \% \mathrm{CI} 1.24\right.$ to $\left.5.14, \mathrm{p}=0.003\right)$. When peak concentric hip abductor strength and peak rearfoot eversion were entered into the forced entry multiple regression model, they explained $47.3 \%$ of peak hip internal rotation $(\mathrm{p}=0.008)$.

For non-excessive rearfoot eversion subgroup, linear regression models demonstrated that peak concentric hip abductor strength significantly predicted peak hip internal rotation during stair ascent $\left(\mathrm{R}^{2}=0.53, \mathrm{~B}=-0.23,95 \% \mathrm{CI}-0.34\right.$ to $\left.-0.12, \mathrm{p}<0.001\right)$; and peak rearfoot eversion did not predict peak hip internal rotation during stair ascent $\left(\mathrm{R}^{2}=0.01, \mathrm{~B}=0.37,95 \% \mathrm{CI}-1.36\right.$ to $\left.2.10, \mathrm{p}=0.65\right)$. When peak concentric hip abductor strength and peak rearfoot eversion were entered into the forced entry multiple regression model, they explained $53.3 \%$ of peak hip internal rotation $(\mathrm{p}=0.002)$.

\section{Discussion}

Our findings indicate that only peak concentric abductor strength explained significantly the variance of peak hip internal rotation during stair ascent in all women with PFP. However, after splitting the participants into subgroups regarding excessive or non-excessive rearfoot eversion, we found that in the excessive rearfoot eversion subgroup, both independent variables (peak concentric abductor strength and peak rearfoot eversion) significantly predicted the variance of peak hip internal rotation during stair ascent. On the other hand, in the non- 
Table 2

Means (standard deviation) subjects' demographics, self-reported measures, kinematics measures and strength measure in PFP participants.

\begin{tabular}{|c|c|c|c|}
\hline Variable & All PFP & Excessive rearfoot eversion subgroup & Normal rearfoot eversion subgroup \\
\hline \multicolumn{4}{|l|}{ Demographics } \\
\hline $\mathrm{N}(\%)$ & $37(100 \%)$ & $18(48.6 \%)$ & $19(51.4 \%)$ \\
\hline Age (years) & $22.00(3.12)$ & $22.05(2.98)$ & $21.96(3.56)$ \\
\hline Body Mass (kg) & $62.02(7.64)$ & $63.04(9.78)$ & $61.12(4.13)$ \\
\hline Height (m) & $1.61(0.59)$ & $1.61(0.89)$ & $1.61(0.50)$ \\
\hline \multicolumn{4}{|l|}{ Self-reported measures } \\
\hline Worst pain level in the last month (VAS) & $51.76(18.86)$ & $56.13(20.01)$ & $47.55(15.20)$ \\
\hline Pain level climbing stairs (VAS) & $14.46(20.16)$ & $18.22(15.99)$ & $11.34(17.14)$ \\
\hline Symptoms duration (months) & $58.43(54.01)$ & $59.17(50.75)$ & $57.12(60.01)$ \\
\hline Self-reported function (AKPS) & $71.32(12.53)$ & $68.12(8.97)$ & $74.98(12.25)$ \\
\hline \multicolumn{4}{|l|}{ Kinematics measures } \\
\hline Cadence (steps/min) & $60.39(7.53)$ & $58.98(8.92)$ & $61.87(6.57)$ \\
\hline Peak hip internal rotation (degrees) & $10.45(10.64)$ & $10.58(7.54)$ & $9.39(8.94)$ \\
\hline Peak rearfoot eversion (degrees) & $6.27(4.76)$ & $8.67(2.46)$ & $2.57(2.48)$ \\
\hline \multicolumn{4}{|l|}{ Strength measure } \\
\hline Peak concentric hip abduction strength ( $\mathrm{N} \mathrm{m} / \mathrm{kg}$ x 100) & $135.50(28.14)$ & $134.03(27.11)$ & $136.59(31.50)$ \\
\hline
\end{tabular}

Abbreviations: VAS = Visual analogue scale $(0-100 \mathrm{~mm})$; AKPS = Anterior knee pain scale (0-100).

excessive rearfoot eversion subgroup, only peak concentric abductor strength significantly predicted peak hip internal rotation during stair ascent.

Generally, peak concentric hip abductor strength explained $27 \%$ of the variance of peak hip internal rotation of women with PFP during stair ascent. Although this finding represents that only a part of the hip alteration is explained by concentric hip abductor strength, it is important to highlight that even slight increases in hip internal rotation angles could result in considerable increases in PFJ stress. As demonstrated by Liao et al. [22], when the hip was internally rotated in $5^{\circ}$ from the natural position, the area of contact between the patella and femur decreased to $22 \%$ and PFJ stress increased to approximately $26 \%$. Therefore, the reduction in PFJ stress could be a possible mediator mechanism through which hip strengthening is a key factor in rehabilitation programs of women with PFP [23]. However, this finding, although significant, reinforces that PFP is a multifactorial condition [3] and cannot be predicted by a single biomechanical alteration. Additional factors which could not be identified by this study may also contribute to excessive hip internal rotation. For instance, a systematic review [24] demonstrated moderate to strong evidence that gluteus medius muscle activity is delayed and of shorter duration during stair ascent in individuals with PFP; this impairment could be related to altered hip motion. There is a need to further explore other reasons by which women with PFP present increased hip internal rotation.

Peak rearfoot eversion did not predict peak hip internal rotation during stair ascent in women with PFP. A reasonable explanation for such finding is that the excessive rearfoot eversion may not be present in all women with PFP. For instance, Noehren et al. [25] compared proximal (hip/trunk) and distal (foot) kinematics between female runners with and without PFP. They found significant differences only in proximal kinematics, suggesting a possible existence of subgroups to explain their findings. Due to multifactorial etiology of PFP, some studies have proposed subgroups regarding different biomechanical alterations, such as excessive rearfoot eversion [21,26]. Selfe et al. [26] proposed three possible subgroups in PFP: 'strong', 'weak and tighter' and 'weak and pronated foot' subgroup. The subgroup composed by individuals who presented excessive rearfoot eversion represented only a portion (33\%) of the entire population assessed. To further explore this possibility, we divided the participants with PFP into two subgroups: (i) excessive rearfoot eversion and (ii) non-excessive rearfoot eversion, based on a cut-off point $\left(4.93^{\circ}\right)$ defined in a recent stair ascent study [21]. Based on this cut-off point, $48.6 \%$ of the participants were classified as excessive rearfoot eversion, which reinforces previous findings $[21,26]$ that this alteration is not presented in the entire population with PFP.

Interestingly, in the excessive rearfoot eversion subgroup, both peak concentric hip abductor strength and peak rearfoot eversion significantly explained the variance of peak hip internal rotation during stair ascent (26\% and $42 \%$, respectively). It seems that lower hip abductor strength is presented in a large portion of PFP population,
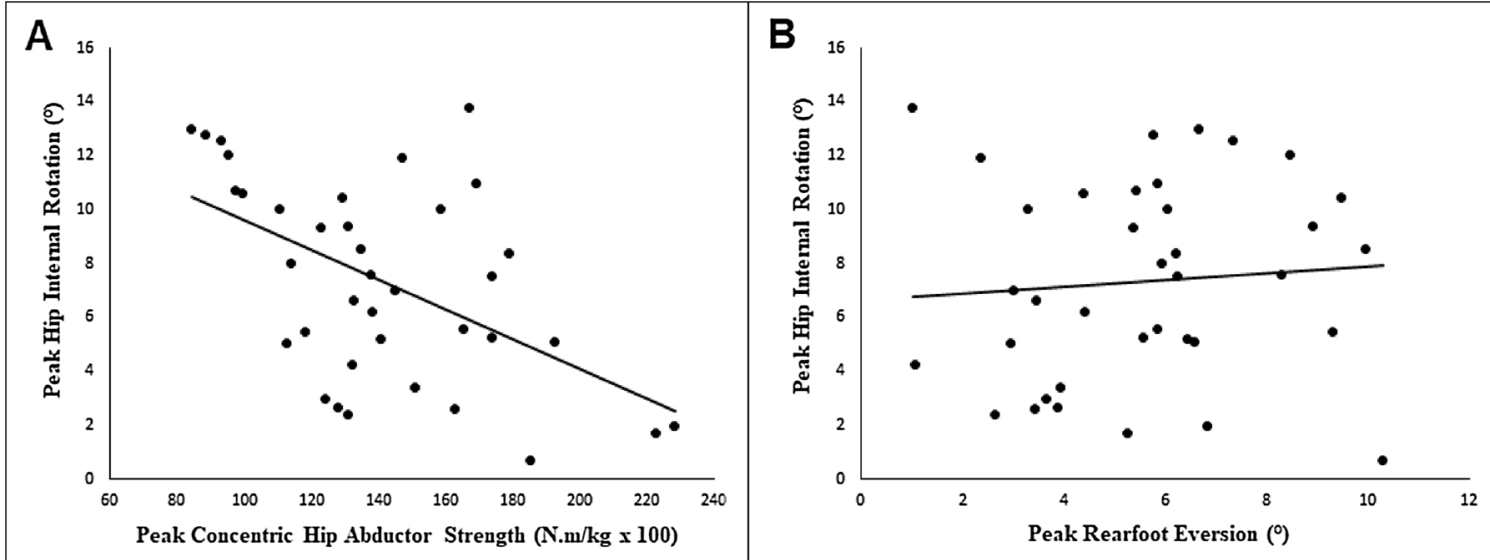

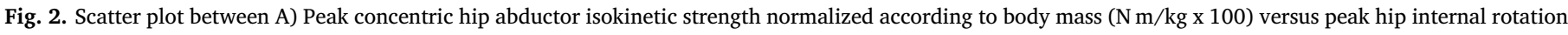
(degrees) in PFP group; B) Peak rearfoot eversion (degrees) versus peak hip internal rotation (degrees) in PFP group. 
therefore, strengthening of hip abductors may benefit most of the individuals with PFP. However, a systematic review [23] investigating the effect of hip rehabilitation for PFP reported that 13 out of 14 studies stated that their rehabilitation programs focused on hip muscle strengthening. Short and moderate term improvements in pain and function were reported following rehabilitation programs targeting hip muscles, but not in long-term. Perhaps, the reason for poor long-term outcomes is the lack of proper muscle strengthening which could be able to change the mechanics of the PFJ, consequently, helping in longterm recovery.

Our findings, in combination with previous results support the idea that a subgroup of women with PFP who also presents excessive rearfoot eversion, is unlikely to benefit from solely hip strengthening. In this direction, Watari et al. [27] identified responders and non-responders after a 6-week muscle strengthening rehabilitation based on kinematics and clinical outcomes. The non-responder subgroup presented greater range of motion in ankle frontal plane when compared to responders, which suggest that interventions focusing on hip muscle strengthening plus interventions targeting distal alterations (i.e., foot orthoses) could be more beneficial for this subgroup [27]. A recent study [28] demonstrated that knee exercises plus foot exercises/orthoses was more effective than solely knee exercises in a subgroup of individuals with PFP with excessive rearfoot eversion. However, positive effects were maintained after 4 months, but not after 12 months. It is important to note that the data collection for this study was conducted when evidences of hip strengthening in individuals with PFP was emerging. Therefore, the incorporation of exercises targeting hip strength could improve the effectiveness of treatment in this subgroup of individuals with PFP. Future randomized clinical trials are needed to investigate the effect of hip strengthening plus foot exercises/orthoses for excessive rearfoot eversion subgroups. Further research using subgrouping approach (e.g. cluster analysis or cut-off points) is required to enhance knowledge about PFP. There is also a need to the developing of strategies to identify subgroups at the initial care, which could help the development of tailored rehabilitation programs. For instance, statistical clustering analyses [26] could be a helpful approach to identify subgroups at the beginning of treatment.

Although peak concentric hip abductor strength and peak rearfoot eversion were found to be predictors of peak hip internal rotation in different subgroups of women with PFP, it is worthy to mention that it does not mean that these impairments are the cause of PFP. This is highlighted by a systematic review [11] reporting a possible discord between prospective and cross-sectional studies. Specifically, lower hip abductor strength was identified in individuals with PFP, but reduced hip strength was not found to be a risk factor for PFP development. Additionally, experimentally induced knee pain in pain-free participants leads to lower strength [29] and altered running and walking biomechanics [30].

\subsection{Limitations}

This study has some limitations that should be acknowledged. Only concentric hip abductor strength was evaluated in our study. Although concentric hip abductor strength is predominantly required to ascending stair, eccentric contractions may also contribute to explain excessive hip internal rotation in women with PFP. Despite we have modified the biomechanical model to enhance the acquisition accuracy of the hip internal rotation angles, these results should be interpreted with caution; as we still do not have technology to determine hip transverse plane movements accurately. Due to the retrospective design used in our study, it is unclear whether a cause-and-effect relationship exist. Therefore, we cannot determine if hip muscle weakness or increased hip internal rotation was present before or after the PFP development. In addition, despite providing some insights about which interventions could be beneficial to women with PFP, the design of this study does not allow intervention prescription based on our findings, but it calls for a randomized controlled trial assessing the effect of foot orthoses plus hip strengthening in a subgroup with excessive rearfoot eversion.

\section{Conclusion}

In general, peak concentric hip abductor strength and not peak rearfoot eversion predicted peak hip internal rotation while ascending stairs. However, in a subgroup with only women with excessive rearfoot eversion (48.6\%), peak concentric hip abductor strength and peak rearfoot eversion predicted the peak hip internal rotation while ascending stairs.

\section{Conflict of interest}

No author has any financial or personal relationship with people or organizations that could inappropriately influence this work.

\section{Acknowledgments}

To São Paulo Research Foundation (FAPESP) for a grant (2014/ 24939-7) and a scholarship (2016/02357-1). The financial sponsors played no role in the design, execution, analysis and interpretation of data, or writing of the study.

\section{Appendix A. Supplementary data}

Supplementary data associated with this article can be found, in the online version, at https://doi.org/10.1016/j.gaitpost.2018.03.037.

\section{References}

[1] P. Kannus, H. Aho, M. Järvinen, S. Niittymäki, Computerized recording of visits to an outpatient sports clinic, Am. J. Sports Med. 15 (1987) 79-85, http://dx.doi.org/ 10.1177/036354658701500112.

[2] M. Boling, D. Padua, S. Marshall, K. Guskiewicz, S. Pyne, A. Beutler, Gender differences in the incidence and prevalence of patellofemoral pain syndrome, Scand. J. Med. Sci. Sports 20 (2010) 725-730, http://dx.doi.org/10.1111/j.1600-0838.2009. 00996.x.

[3] K.M. Crossley, J.J. Stefanik, J. Selfe, N.J. Collins, I.S. Davis, C.M. Powers, J. McConnell, B. Vicenzino, D.M. Bazett-Jones, J.-F. Esculier, D. Morrissey, M.J. Callaghan, Patellofemoral pain consensus statement from the 4th International Patellofemoral Pain Research Retreat, Manchester. Part 1: terminology, definitions, clinical examination, natural history, patellofemoral osteoarthritis and patient-reported outcome m, Br. J. Sports Med. 50 (2016) (2016) 839-843, http://dx.doi.org/ 10.1136/bjsports-2016-096384.

[4] D. De Oliveira Silva, C.J. Barton, M.F. Pazzinatto, R.V. Briani, F.M. De Azevedo, Proximal mechanics during stair ascent are more discriminate of females with patellofemoral pain than distal mechanics, Clin. Biomech. 35 (2016) 56-61, http://dx doi.org/10.1016/j.clinbiomech.2016.04.009.

[5] N.E. Lankhorst, M. van Middelkoop, K.M. Crossley, S.M.A. Bierma-Zeinstra, E.H.G. Oei, B. Vicenzino, N.J. Collins, Factors that predict a poor outcome 5-8 years after the diagnosis of patellofemoral pain: a multicentre observational analysis, $\mathrm{Br}$. J. Sports Med. (2015) 1-7, http://dx.doi.org/10.1136/bjsports-2015-094664.

[6] C.M. Powers, The influence of altered lower-extremity kinematics on patellofemoral joint dysfunction: a theoretical perspective, J. Orthop. Sport Phys. Ther. 33 (2003) 639-646, http://dx.doi.org/10.2519/jospt.2003.33.11.639.

[7] C.M. Powers, S.R. Ward, M. Fredericson, M. Guillet, F.G. Shellock, Patellofemoral kinematics during weight-bearing and non-weight-bearing knee extension in persons with lateral subluxation of the patella: a preliminary study, J. Orthop. Sport Phys. Ther. 33 (2003) 677-685, http://dx.doi.org/10.2519/jospt.2003.33.11.677.

[8] C.M. Powers, E. Witvrouw, I.S. Davis, K.M. Crossley, Evidence-based framework for a pathomechanical model of patellofemoral pain: 2017 patellofemoral pain consensus statement from the 4th International Patellofemoral Pain Research Retreat, Manchester, UK: part 3, Br. J. Sports Med. (2017), http://dx.doi.org/10.1136/ bjsports-2017-098717 (bjsports-2017-098717).

[9] C.M. Powers, The influence of abnormal hip mechanics on knee injury: a biomechanical perspective, J. Orthop. Sport Phys. Ther. 40 (2010) 42-51, http://dx. doi.org/10.2519/jospt.2010.3337.

[10] D. Tiberio, The effect of excessive subtalar joint pronation on patellofemoral joint mechanics: a theoretical model, J. Orthop. Sport Phys. Ther. 9 (1987) 160-169.

[11] M.S. Rathleff, C.R. Rathleff, K.M. Crossley, C.J. Barton, Is hip strength a risk factor for patellofemoral pain? A systematic review and meta-analysis, Br. J. Sports Med. 48 (2014), http://dx.doi.org/10.1136/bjsports-2013-093305 1088-1088.

[12] R. de M. Baldon, T.H. Nakagawa, T.B. Muniz, C.F. Amorim, C.D. Maciel, F.V. Serrão, Eccentric hip muscle function in females with and without patellofemoral pain 
syndrome, J. Athl. Train. 44 (2009) 490-496, http://dx.doi.org/10.4085/1062 6050-44.5.490.

[13] M.C. Boling, D.A. Padua, R.A. Creighton, Concentric and eccentric torque of the hip musculature in individuals with and without patellofemoral pain, J. Athl. Train. 44 (2009) 7-13, http://dx.doi.org/10.4085/1062-6050-44.1.7.

[14] B. Tyldesley, J. Grieve, Muscles, Nerves and Movement in Human Occupation, 3rd ed., Wiley-Blackwell, 2002.

[15] P. DeVita, J. Helseth, T. Hotobágyi, Muscles do more positive than negative work in human locomotion, J. Exp. Biol. 210 (2008) 3361-3373, http://dx.doi.org/10. 1242/jeb.003970.Muscles.

[16] C.J. Barton, P. Levinger, K.M. Crossley, K.E. Webster, H.B. Menz, The relationship between rearfoot, tibial and hip kinematics in individuals with patellofemoral pain syndrome, Clin. Biomech. 27 (2012) 702-705, http://dx.doi.org/10.1016/j. clinbiomech.2012.02.007.

[17] J.F. Esculier, B. Dubois, L.J. Bouyer, B.J. McFadyen, J.S. Roy, Footwear characteristics are related to running mechanics in runners with patellofemoral pain, Gait Posture 54 (2017) 144-147, http://dx.doi.org/10.1016/j.gaitpost.2017.03. 010.

[18] D. De Oliveira Silva, R.V. Briani, M.F. Pazzinatto, D. Ferrari, F.A. Aragão, C.E. de Albuquerque, N. Alves, F.M. de Azevedo, Reliability and differentiation capability of dynamic and static kinematic measurements of rearfoot eversion in patellofemoral pain, Clin. Biomech. 30 (2015) 144-148, http://dx.doi.org/10.1016/j. clinbiomech.2014.12.009.

[19] D. De Oliveira Silva, F.H. Magalhães, M.F. Pazzinatto, R.V. Briani, A.S. Ferreira, F.A. Aragão, F.M. de Azevedo, Contribution of altered hip, knee and foot kinematics to dynamic postural impairments in females with patellofemoral pain during stair ascent, Knee 23 (2016) 376-381, http://dx.doi.org/10.1016/j.knee.2016.01.014.

[20] K. Jordan, J.H. Challis, K.M. Newell, Walking speed influences on gait cycle variability, Gait Posture 26 (2007) 128-134, http://dx.doi.org/10.1016/j.gaitpost. 2006.08.010.

[21] D. Ferrari, R.V. Briani, D. de Oliveira Silva, M.F. Pazzinatto, A.S. Ferreira, N. Alves, F.M. de Azevedo, Higher pain level and lower functional capacity are associated with the number of altered kinematics in women with patellofemoral pain, Gait
Posture 60 (2018) 268-272, http://dx.doi.org/10.1016/j.gaitpost.2017.07.034.

[22] T.C. Liao, N. Yang, K.Y. Ho, S. Farrokhi, C.M. Powers, Femur rotation increases patella cartilage stress in females with patellofemoral pain, Med. Sci. Sports Exerc. 47 (2015) 1775-1780, http://dx.doi.org/10.1249/MSS.0000000000000617.

[23] S. Lack, C. Barton, O. Sohan, K. Crossley, D. Morrissey, Proximal muscle rehabilitation is effective for patellofemoral pain: a systematic review with metaanalysis, Br. J. Sports Med. 49 (2015) 1365-1376, http://dx.doi.org/10.1136/ bjsports2015094723.

[24] C.J. Barton, S. Lack, P. Malliaras, D. Morrissey, Gluteal muscle activity and patellofemoral pain syndrome: a systematic review, Br. J. Sports Med. 47 (2013) 207-214, http://dx.doi.org/10.1136/bjsports-2012-090953.

[25] B. Noehren, M.B. Pohl, Z. Sanchez, T. Cunningham, C. Lattermann, Proximal and distal kinematics in female runners with patellofemoral pain, Clin. Biomech. 27 (2012) 366-371, http://dx.doi.org/10.1016/j.clinbiomech.2011.10.005.

[26] J. Selfe, J. Janssen, M. Callaghan, E. Witvrouw, C. Sutton, J. Richards, M. Stokes, D. Martin, J. Dixon, R. Hogarth, V. Baltzopoulos, E. Ritchie, N. Arden, P. Dey, Are there three main subgroups within the patellofemoral pain population? A detailed characterisation study of 127 patients to help develop targeted intervention (TIPPs), Br. J. Sports Med. 50 (2016) 873-880, http://dx.doi.org/10.1136/bjsports-2015094792.

[27] R. Watari, D. Kobsar, A. Phinyomark, S. Osis, R. Ferber, Determination of patellofemoral pain sub-groups and development of a method for predicting treatment outcome using running gait kinematics, Clin. Biomech. 38 (2016) 13-21, http://dx. doi.org/10.1016/j.clinbiomech.2016.08.003.

[28] C.M. Mølgaard, M.S. Rathleff, J. Andreasen, M. Christensen, S. LundbyeChristensen, O. Simonsen, S. Kaalund, Foot exercises and foot orthoses are more effective than knee focused exercises in individuals with patellofemoral pain, J. Sci. Med. Sport 21 (2017) 10-15, http://dx.doi.org/10.1016/j.jsams.2017.05.019.

[29] M. Henriksen, S. Rosager, J. Aaboe, T. Graven-Nielsen, H. Bliddal, Experimental knee pain reduces muscle strength, J. Pain 12 (2011) 460-467.

[30] M.K. Seeley, J. Park, D. King, J. Ty Hopkins, A novel experimental knee-pain model affects perceived pain and movement biomechanics, J. Athl. Train. 48 (2013) 337-345, http://dx.doi.org/10.4085/1062-6050-48.2.02. 